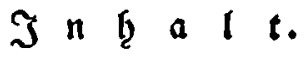

A I E Einle itung, umtiß ciner Ablandlung von Der Einbeit Der romanijhen und germanijchen Bolfer und von ibrer gemeinfdaftliden Eutwides lung.

- S. xvII

(5rite $\mathfrak{B}$ ud).

Erfes Eapitel. Lage von Frantrich und yon Italien. Starls VHI. 3ug uach Reapel. • S. 3

1. Jrantrcid). Die Eapetingen, 5. 3. - Eond XI., 3. - Faris VIII. 2Infang, s. - 3uftand von Srane: seid, 7. - 2ubitat auf Reapel, 9. - auf Эeru= falem, 10. - Rúftung. Raris Ratur, 11 .

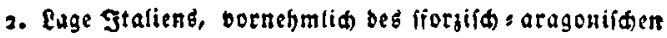

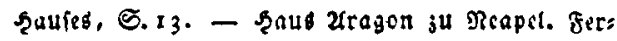
rantes erfer Frieg, 13. - Biderung, 14. 2lifonio, 25. - Blociter Rrieg, 16. - Reue Eiфerung, 17. - Salus Eforja uno Lodobico, 18. - Eorenzo Dei Mesici unto Snnoceng YILI., 22, Zagemeinere Betradtung, 23.

Epaltung pes fiordif : aragonifiden 5̧aufes, 25. - Mes Dici für Zragen; Pabfr Zllerander VI. fár dic Bfors 3en, 26. - Ende Eertanted, 28. - 2llesander ju zllfon: 10 II., 30. - Ftalienilder Arieg. 2ungriff 2lfon: $10 \%$ auf Eovobiso, 30 .

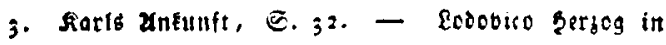
Manland, 33. - Kail th Zoscana, 35. - 23: Der alepaltber V1., 40. - ZBiber alfoulo, 42. - 
21fonio's 26odantung uno Fludt, 43. - Frieg zwifhen farl'n und zerrantin, 44 .

3 wente Gapitel. Pon ber Srúndung Spas

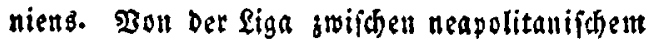
und maylánbijhem Intereffe. \$on Dem istrieg in Manland und Neapel 1405. 1496. . . . S. 48

x. Epanien - Faftitien in Barwirtung, 5.48 . 2ragon in Berroirrung, 49. - Bende vereinigt.

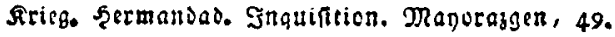
- Reue Roniggrmatt, s3. - Ridtung gegen dic unglduvigen, s4. - Entbedung von 2merifa, 55 .

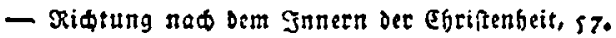
- 2uf Peapel, 58. - Bru申 mit RarIn, 59.

2. Eodobico's Gefabe und feine nd 5. 60. - Eiga grifhen den neapolitonifden uno Den manlandifwen 3erburieten, 62.

3. Der Turfengug eufgegeven, ఠ, 63. - Dronungen in Neapel, 64, - Notjug Raris, 69.

4. Dawegungen tor und binter ifst, 6. 66. - Sort nad) der Romuardey, 68. - Edratit ven forno: Do. Fidfeff Ferranting nod Reapel, 70.

5. Der Serieg bon Mobera und die Bictereroberung von neapel, 5. 74. - Eroberung ber Stabt un১ Der Zeftefie, - ber zucugzen - der eefter Rergebes ne bor Ealabricn, 77. - Eieg im Diten nflo auf Det amenten Bergebene vor Ealabrien, 78. - Ent: idcidung in ber Mitte bes Bebirgs, 80. - Ferran: tin's Zor, 82. - Eage der Parthenen, 83.

Drittes Eayitel. Marimilian auf Dem Reich: tag gu Borms und in Stalien. wiberftall bef Florentiner. Sieg Der Liga. . . .

Eage und 2lbitten Marimilians, S. 84. - Eeitte Nas ๑. 84 tur, 86. - Reidstag 6น 2Borms, 89. - 24uss

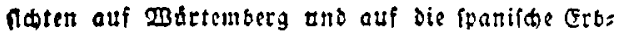
f6aft, 89. - Doppette fertegbratt ber Deutigett, 90. - Worauf das Retá beruffe, 91. - Defreid

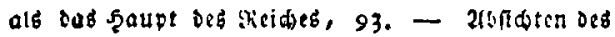

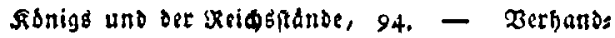


Iungen bendes, 96. - Dic biet Eintigtungen bies ies Reiditags, 97.

Ridtung Rarimilians auf Ttalien, 99. - Kondten bon Benedig und sobcrics, 100. - Zujaminatunft Eos covicu's ans Tiariaitiatts, roo. - Berfall ser belts

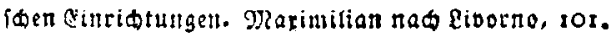
Elorentiner. SGre Madt, 5. 103. - Shre Beiddftigun, get, 104. - Eavenarda, 106. - Balia. Stre 2tufs 10jung, 109. - Propolate 3erfañung im 3unn 2495 , x:0. - Miserftond gegen Marimilian, Eieg dee Fiortutinet, 1 rt. - Egrinlide sRidtung, 113. Neuer Famp mit Dem Pabit, I 4 4. - Defien fris

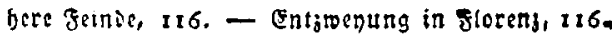
- Entibcidnng, 120. - Z0D Roris VIII, 121. - Tod Savoracola's, 122.

Grobe Bunde bon Spanten, 123. - Mit England. Şęins

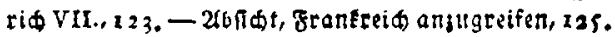
- Erbebing Marimilians DurG 20uttemberg, 126 .

Niertes Capitel. Hutergang bes fortijas saras gonijifen Sautes. . . . .

2ngrif Rarimifians uno 3erfall ber Riga, 5. 229. Eubrig des 3rostften Ratur und 2rnfang, t3o. Radutu auf Manland uno Peapel, 133. - Bene:

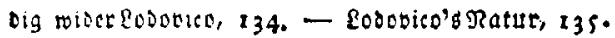
- Borauf er traute, 136. - Darimilian mit Eodobico, 137. - ablidt Der Franzoien wider Ben: De, 138. - 3wift zrifhen Zyrol und Graubinden, 139. - Sirieg ber Edweiz wiber Rarimilian, 140. - Buno der Edwits mit \{uowig, r42. - Sd)lad, ten am Shraderló und bey Jrafen, 143. -

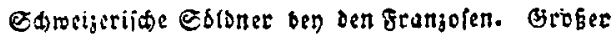
Arieg JRarimilian,, 146. - Prieg Det Eranjofen wider Eosobito, 149. - Reue Geftalt Ftaticn, 154. - 3wenter Frieg Eobobico's nat Dem fries ben in Deutidland, xs6, - Wiesertunft der Bfor: jen, I59. - Entideidung ourd die Edweizer, 162.

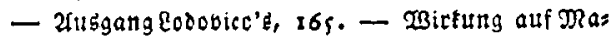
rimilian, 168. - Der Pablt wider feine 3 afallen, 16\$. - 2Bider Smola und forli, 173. - 2Bider

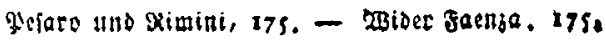


- Míggeidid ber 2ragorten, 177. - Betradiung, 181. - Frteg oer Jenetianer mit ben Tarten uno ßernando's mit den Jiauren, 283 .

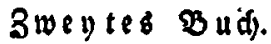

Einleitung.

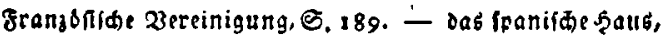
1.91. - NRatbmaglide Berbindung Bender, 193.

\section{Erfes capitel.}

1. Der erfte Strieg in Seapel und Romagna bis auf Allefanders $\mathfrak{T o D}$. S. 195

Urfaden bes frieges, 195. - Reapolitanifiter frieg bom Juny 1502 bis jum Jankar 1503 . Fitter: thum, 196. - - Mumagner Frieg ju berielven 3it. Definen, 20x. - Slitider Erfolg venter Fi iege, 207. - Dendung Der Dinge, 207, In Meapel, 207. - 3win Frldidladten, 209, - auÆgung, 211. - खBendung in şinfiøt auf der Pabt, 212. - Tod 2lleranders, 213.

2. Bwenter Rrieg in Reapel uno Romagna bis auf Eefars Ausgang.

3erwirrung in Rom und Romagna, S. 219. - Pabit: roubl. Pius III, 216. - Gulius II. Yauft, 217. - Srieg in Roufitlon, 218. - 2lm Grariglian, 219. - Eiderung und Pidne Bonsal's, 220. - Benetianer in Romagna. 24uggang ङefars, 221.

\section{Bweytes capitel.}

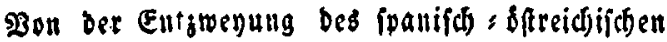
Staujes. • • • . .

Eingang. Beranlafinti, E. 225.

1. SRaximilian Durdh Die Einwirfung bes fran: Isfifchen Bundes Gieger und 5arr in Deutfhlatt.

Derfau Des Kegimentes. Orúnoung unt Betfall ber 
Efurfurfentage, E. 227. - Die Bahe ton Batirnlandifut, 229. - Doppelter Frieg, 23x. - In Der Pfal, s31. - In Baiern, 231. - Woufommener Cieg des Şaufes Defts re(t), 233 .

2. Mlane und Erfolg Des Saujes Deftreich.

S.235 Fldne ju einer Uniberialntonardie, 5: 235. erfees Sindernif. Eudwig und Ferditand in 3und, 237. - Sุaủ Deftreid mit Jingtand, 240. - 5âu Ocfrecid in Saftilien, 24x. 5̧aus Deftrcid in ungarn, 244. - Baiber Stulien, 246. - Entjdeti endeg Şindernif, 248.

3. Gerbinand Serr in seapel und Caftilien. . ஒ. 248

Conzul und darauf gerdinano in Reapel, 厄. 248. - Suana, Die Parthenen, Kimené, endid Fetdinath in Caftutien, 252.

4. ARubere Unternebmungen Ferbinands.

5. 250 Drittes sapiter.

Bou Benedig und Sulius II,

5.264

1. Sanbel, Eroberungen, Berfaffung Der $\mathfrak{B e s}$ netianer; Angriff auf bie Romagna.

S.264

Bonft und bamalg, E. 264. - Deftlider Şandel, 265. - Weftider Şandet, 266. - Şan: Delsgefebe. 267. - Eroberungen, 267. Bur See 1388 - 1420, 267. - Uno ferner, 268. - In Stalien, 268. - 9josenigo uno Fogcari, 269. - Bermuthung von iffer $\mathfrak{Z}$ fonung, 270 .

2. Julius des Bwenten erferbaten unb boppelte Abficten. . . . . . .

Eintıwenung Fultus mit Benedig, 5. 273. - Sha: rafter bes Pabfes, 274. - Bentiooglio bu 2Bos Iogna, 275. - Julits wider ifn, $375 .-$ Sulius in perugia, 276. - in Bologna, 276. - 5inderniffe weiterer abfidstell. Revolution ill Genua, 278. - brointe 2(biid)t ocs Pabs fiet, 281 . 
3. Entoratungen ber Partugiefer. Itmidilag bes venetianifiben şanbels.

Die Mrauren, D. i. Zenber, Prongoten uno andere

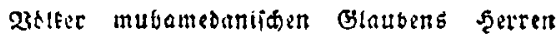
Dis indilan şandels, 5. $28 \mathrm{I}$. - Befagr fúc Liefen Şandel, 283. - Don Nranuel, 284. - Entbertung ber unvetantten Rifte, 284. Bagco in Ratitut, 285. - Deut uniernets: mungert, 286. - Erfter Rtivg, 2Bider Den $3 a=$ storin, 28\%. - 3ronter Frieg. 2Bidar 9n: dianer und intianitide Mrauren, 288 . - Bers anderung des f̧andels, 292. - Borberettuth 3uth oritten frrieg, 293.

4. Angriff Maximilians. Silsung Der Siga von Cambray wider die Eroberuitgen dir $3 e n e s$ tianer.

Birureitete Borftellung von Benedig, ๔. 294. Marimilians 2undat miser grantreid, 295. Ridtung grarinilland wiber Benedig, 296. ulmidlug. 297. - Ceroverungen det Benctin: ner áber Den Ratier, 299. - Buno von Cams bray, 302.

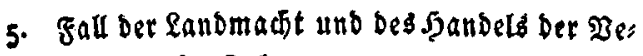
netianer im $J_{a b r} 1509$.

Eage Der Dinge, 5. 304. - Syeffungen, Ruftut: 6. 304 gen Benedigs, 306. - Sirieg, 308. - Edtaqte bon Gfiara d'2dda, 2aila ober Jgandeub, 3 ro. - Erforg, 312. - Entiकeiduttg in Fndient, 314.

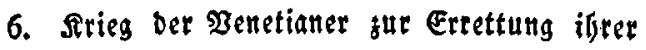
Stadt und sines ₹lyeiles ber Landfchaft. Neuer Rrieg ourch Rónig und Raifer, S. 316.

7. Die Untermebmungen bes Pabfies zur $\mathfrak{3 e s}$ frevung Italiens. • • .

Pidtung des $\mathfrak{Y a u f t e s}$ mider \&ubsig, E. 322. - ro: Der ferrara, 324. - Erfte Berfude, 324. - 
Unftalt zu jwenten, $325 .-$ Rage der Є由mei= 1er, 326. - 3reptet Berfud, 328. - Sulius in Bologne angegrifien, 331 . - Dritter Ber: fu\$ bes Pabfes, 334. - Einfolt, 337. -

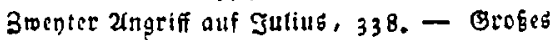
Mígerdice des Pabfes, 339, - Reuer Bers fall Der benetianifoen Dinge, 340 .

SڤIuß Des Eapitels.

S. 342

Biertes Eapitel.

Die Erbebung Des ipaniid : Sfereichichen Saufes bis nabe zur godjaten Beralt in Europa.

I. Sulius im Bund mit Epanten. . . 5.346 antandigung zrener Concilien, อ. 346, - 3uno Julitt nit Jerdinand, 347. - Die Edrecizer fúc ñd felbft in Frieg, 348. - Reucr srieg Des Pabres. Eein bierser Beriud, 351. Zngriff Der Gpanier unb Bentianer, 354.

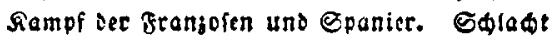
ben Raviuna, 357 .

2. Biloung cinet neuen liga. Lage und Eintritt Englande. . . . . .

şeintids VIr. Zntutunt und intere Maakresetn,

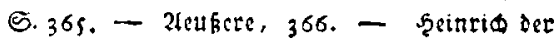
216te, 367, - Bentritt şzinriøs uto Maxi= miliatt su dem 2iund. Berpinigung und $266=$

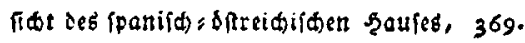

3. Eroberung von Manlatib. . - cigentide

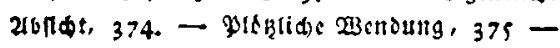
Mranland in ben şanden der equetizer, 376. - Das (paniia) şeer rist detrud vor, 378.

4. Eroberung von Ravarra. 
rens, Pifa, Concitium, 383. - Eharatter Sos hant's bei Medici, 389. - Medici Durd Cars Dena unb Earg in Florem, 387.

6. Fernere abfihten unb, Etfolge. - •

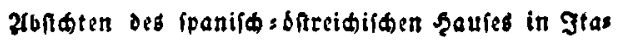
lict, 5.391. - Rage, zod des Paufies Stus Itus, 391. - Dritter Etfoly defielben Şaules. Die Wabl Seo's des Befnten, 393.

7. Sambf Der Franjoien und Echweifer um IRaps land. • . • . • • ङ. 394

3wiidengeididte. Eage der Bdwrizer, E. 394. Lnterbanolungen uno Rrieg mit Eubrig XII., 396. - Cकlat) ben Rovara, 400.

8. Brober ferieg wiber franfreid und $\mathfrak{B e t u e b i g . ~}$ Bergleidung, E. 403. - 2ngriff von biet sectet! auf sranferi申 und 23 nedig, 403. - Srangor fen unb Edotten, 409. - Groke Gifafe bon Franferid, 413. - Kettung, Qber farker 3ers luft, 413. - Misgeftid bon Benedig, 415. - Sieg bon Defterid: Epanien und weitere aunchten auf biefem $28 \mathrm{sg}, 416$.

Befflus des vierten Eapitels und des jweoten \$udb6. - . . - . -

Gang neucr plane bis nabe an ifre Bollenoung im

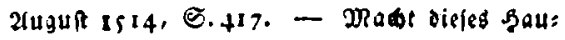
ier in Europo und amerifa, 420. 